\title{
Branch pulmonary artery Doppler in the assessment of atrial restriction after the hybrid procedure for hypoplastic left heart syndrome
}

\author{
Hannah Bellsham-Revell ${ }^{1}$, Eva Kapravelou ${ }^{1}$, Shane Tibby $^{1}$, Kelly Peacock ${ }^{1}$, and John \\ Simpson ${ }^{1}$ \\ ${ }^{1}$ Evelina London Children's Hospital
}

January 17, 2021

\begin{abstract}
Introduction: The hybrid procedure (HP) - bilateral pulmonary artery bands+ductal stent - is an alternative palliation for high-risk hypoplastic left heart syndrome (HLHS) patients. Assessment and management of atrial restriction here is debated and we sought to identify useful echocardiographic parameters. Methods: Patients undergoing HP for HLHS were identified. Echocardiograms at birth, immediately after HP and pre-and post-atrial intervention were reviewed as well as time-matched echocardiograms in HP patients not requiring atrial intervention. Atrial septal parameters and systolic (Svel), diastolic (Dvel) and mean velocities were recorded on both bands and systolic to diastolic velocity ratio (Svel:Dvel), pulsatility index (PI) and velocity time integral (VTI) calculated. Results: Fifteen HP were compared to 5 controls. Age effect was not significant. There was a significant rise in peak Svel and changes in Dvel, Svel:Dvel and PI over time $(<0.05)$. Although the pattern of change similar, the fall in Dvel and rise in Svel:Dvel and PI was more significant on the left. Compared to controls, transatrial gradient and VTI were significantly increased initially post-hybrid (median $0.4 \mathrm{~m} / \mathrm{s}$ vs $0.7 \mathrm{~m} / \mathrm{s} \mathrm{p}=0.04 ; 29.1 \mathrm{~cm}$ vs $64.6 \mathrm{~cm} \mathrm{p}=0.018$ ) but not significantly different prior to septostomy when the only significant differences were seen with all left band parameters. Conclusions: After HP the transatrial gradient alone cannot be used. Significant changes in band Doppler parameters were not apparent in the control group but were principally seen in the left band with reduction in Dvel and increase in Svel:Dvel and $\mathrm{PI}$ in those undergoing an atrial intervention.
\end{abstract}

\section{Introduction}

The hybrid procedure (HP, bilateral pulmonary artery bands and stenting of the arterial duct) for hypoplastic left heart syndrome (HLHS) and its variants was proposed in the early 1990s(1) as an alternative palliation to the Norwood procedure, particularly to avoid cardiopulmonary bypass in high-risk infants. Atrial septectomy forms part of the standard Norwood procedure, whereas during the hybrid procedure any atrial intervention will usually be performed off bypass. There is varying opinion on the optimal timing of atrial intervention in patients with HLHS undergoing HP from at the time of the procedure to performing later if signs of atrial restriction(2-4). Decisions about whether or not to intervene on the atrial septum are generally based on echocardiographic assessment including mean gradient across the atrial septum, left atrial size and pulmonary vein Doppler interrogation (5). These parameters may be abnormal in HLHS (6) and are impacted by high pulmonary blood flow after initial palliation. Such assessment is clinically important to optimise free flow of oxygenated blood from the left atrium to the systemic arterial circulation and to avoid severe left atrial hypertension with potentially deleterious effects on development of the pulmonary vasculature.

Fenstermaker at el(5) described changes in the pulmonary artery band Doppler over time following HP. That study described alterations in the shape of the Doppler trace in a small subset of patients who required atrial septal intervention. In this retrospective study, we review the characteristic of the branch pulmonary 
artery band Doppler values in patients with HLHS after the hybrid procedure, who have required atrial septal intervention prior to their second palliation. We hypothesized that the previously seen changes in pulmonary artery band Dopplers would aid in identifying patients who require atrial septal intervention. Patients were divided into two groups (critical aortic stenosis and classical HLHS) to further investigate the potential impact of residual forward flow through the left heart.

\section{Methods and Materials}

Ethical and institutional approval was obtained. In our institution, hybrid palliation is performed in three main groups: a) infants [?] $2.7 \mathrm{~kg} \mathrm{~b}$ ) poor pre-morbid condition or with significant risk factors for Norwood Procedure (significant tricuspid regurgitation, right ventricular dysfunction, neurological or multi-organ dysfunction after presentation) and c) 'borderline' left heart structures where biventricular repair was felt to be possible in the future.

Via a median sternotomy, pulmonary artery bands fashioned from a cut section of Gortex shunt are placed around each branch pulmonary artery and secured. A sheath is then introduced directly to the pulmonary artery and the stent deployed under fluoroscopic guidance. Our usual practice is not to perform an atrial septal intervention at the time of HP unless there is evidence of atrial restriction prior to the procedure as defined by a high mean atrial gradient with A wave reversal on pulmonary venous Dopplers along with clinical evidence with chest $\mathrm{x}$-ray congestion and low saturations.

After HP, patients are assessed on an individual basis. In this era according to surgeon preference, some will undergo a Hybrid to Norwood conversion procedure and then a later hemi-Fontan (HF), or if deemed unsuitable for an early Norwood (e.g. aberrant right subclavian artery, significant tricuspid regurgitation or impaired right ventricular systolic function), may undergo a comprehensive second stage (CSII) consisting of a Damus-Kaye-Stansel anastomosis, reconstruction of the aortic arch and HF at around 6 months of age.

\section{Patient Selection}

Patients with HLHS who had undergone HP with one or more further interventions on the atrial septum, prior to the second palliation, were identified from the departmental database (HeartSuite). Patients with atrioventricular and ventriculoarterial concordance with mitral and aortic stenosis or atresia, with no significant ventricular septal defects were included. Patients with other variants such as unbalanced atrioventricular septal defects or double outlet right ventricle were excluded. Patient demographics along with the date of procedures were recorded. Further procedures and current status were recorded from the same departmental database.

Echocardiograms were identified, reviewed and analysed on the Philips Xcelera workstation. Time-points for analysis were:

- Within 24 hours of hybrid procedure

- Prior to atrial septal intervention

- Post atrial septal intervention

\section{Control Group}

A control group of patients with left heart hypoplasia (as defined above) who had undergone HP but with no subsequent atrial septal intervention were also included. Initial post-hybrid echocardiogram was reviewed as well as an echocardiogram around one month of age or as close in time to this as possible.

\section{Echocardiographic Assessment}

Most echocardiograms were performed on a Philips IE33 system (Philips Medical Systems, Andover, Mass. USA) with an age and size appropriate probe (S8 or S12). The small remainder were performed on GE Vivid Q (General Electric Corporation, Milwaukee, USA) with an age and size appropriate probe. Atrial septal Doppler assessment was performed from a subcostal view; pulmonary artery band Doppler interrogation were usually obtained using a high parasternal view and interrogated with continuous wave Doppler. 
On each of the echocardiograms, the atrial septal Doppler along with each pulmonary artery band Doppler was reviewed. The atrial septal Doppler wave was traced over two cardiac cycles to calculate the mean transatrial gradient (IAS) (Figure 1). The pulmonary artery band Doppler for the right (RPA) and left (LPA) pulmonary artery bands was traced over two cardiac cycles. The mean velocity and VTI were measured as well as the peak systolic velocity ( $\mathrm{S}$ vel) and diastolic velocity (D vel) (Figure 2). The systolic to diastolic velocity ratio ( $\mathrm{S}$ vel: $\mathrm{D}$ vel) was calculated, along with the pulsatility index ((systolic velocity minus diastolic velocity) divided by the mean velocity, PI). Additionally, the shape of the traces was recorded as either sawtooth or pulsatile, along with the presence of any notching of the descent (Figure 3).

One operator performed all measurements (HBR). Intra-user variability was tested by the same operator measuring the parameters on 5 echocardiograms $>3$ weeks after the initial measurements. Another operator (EK) measured the parameters on the same 5 echocardiograms to assess inter-user variability.

\section{Statistics}

Differences in the atrial septum and pulmonary artery band parameters were assessed using the paired student t-test and differences between the two groups (AS and HLHS) was assessed using the independent student t-test. Inter- and intra-user variability was assessed using the intraclass correlation coefficient. Statistical analysis was performed using Stata version 14.1 (StatCorp, Texas). Change in variables over time was modelled using multilevel mixed effects linear regression, with time modelled as a categorical variable. All models included patient age as a covariate. Change over time was expressed using $3 \mathrm{P}$ values: time, group (left versus right) and group-time interaction (see table 3 legend for further explanation).

\section{Results}

From December 2005 (start of the hybrid program at this institution) to May 2015, 20/66 (30.3\%) patients with left heart hypoplasia underwent an atrial intervention after HP. Two patients in the early era were excluded as their echocardiographic data was not available, two were excluded as they underwent a septal intervention at the time of HP, and one was excluded as they underwent a biventricular repair. Six patients had critical aortic stenosis with forward flow through the left heart, and 9 had classical HLHS. Five control patients were included. The patient characteristics are shown in table 1.

Eleven patients had a septal intervention as the sole intervention. Three had additional intervention on the ductal stent (one patient had a septostomy due to concern over gradient and two as it was felt septostomy should be performed during the catheter) and one had an aortic valve balloon (septostomy was performed as the gradient was $34 \mathrm{mmHg}$ with clinical evidence of elevated left atrial pressures). Pre-procedural invasive gradient was measured in 7 patients, median (range) $9 \mathrm{mmHg}(4-34 \mathrm{mmHg})$.

\section{Inter and Intrauser variability}

Intra-user variability for all parameters was $>0.787$ and $>0.772$ for inter-user variability, falling into the good range.

Changes in band parameters over time (tables 2 and 3)

In patients who required an atrial intervention there was a significant increase in systolic velocity (figure 4a) with time and a significant decrease in diastolic velocity prior to septostomy, with a rise post-septostomy (figure $4 \mathrm{~b}$ ). with time The S vel: D vel and PI also showed a significant change over time, with an increase prior to septostomy and then a fall after the septostomy (figure $4 \mathrm{~b}$ and $4 \mathrm{c}$ ). There were no significant differences in the pattern of change of parameters over time between the two bands but the response of the left compared to the right pulmonary artery band Dopplers in respect to diastolic velocity, S vel: D vel ratio and PI was more significant (figure 4a-d).

Prior to septostomy compared to controls (table 4$)$, the LPA S vel was significantly lower $(2.5 \mathrm{~m} / \mathrm{s}$ vs $3.5 \mathrm{~m} / \mathrm{s}$, $\mathrm{P}<0.05)$ as was the LPA D vel $(1.1 \mathrm{~m} / \mathrm{s}$ vs $1.6 \mathrm{~m} / \mathrm{s}, \mathrm{p}<0.05)$ with a significantly lower mean $(1.8 \mathrm{~m} / \mathrm{s}$ vs $2.6 \mathrm{~m} / \mathrm{s}, \mathrm{P}<0.05)$ and VTI $(144.5 \mathrm{~cm}$ vs $196.7 \mathrm{~cm}, \mathrm{p}<0.05)$. The LPA $\mathrm{S}$ vel: D vel ratio and PI were also 
significantly higher (2.8 vs 2.0 and 1.0 vs 0.7 respectively, p $<0.05$ ). There were no significant differences in septal mean velocity or VTI or RPA parameters.

The shape of the RPA and LPA Doppler was predominantly "saw-tooth" in most patients (97\%). It was noted that in the LPA this pattern changed to a more pulsatile pattern (reflecting a lower diastolic) in several of the patients who required an atrial intervention. Even on the immediate post-intervention scan in some patients, there was an improvement in the shape back to "sawtooth" (figure 1).

\section{Discussion}

The recognition of need for atrial septal intervention after HP remains relatively controversial, with little evidence to support a consistent approach when there is such wide heterogeneity of the interatrial communication (2-4). It is well recognised that initially unrestrictive interatrial communications may become increasingly restrictive, and also that a degree of atrial restriction may be acceptable, and indeed important, for those where biventricular repair may be possible in the future (7). It is an important decision as if a child is well-balanced even in the face of flow acceleration seen on echocardiography, they may become less well balanced after a septal intervention.

There are many factors affecting echocardiographic assessment of the atrial septum in HLHS. A colour and 2D approach will highlight aliasing, but this can be present even with a generous communication in the context of high pulmonary blood flow. Sometimes application of the pulmonary artery bands may reduce the pulmonary flow sufficiently to reduce the perceived transatrial gradient. Pulmonary vein Dopplers were not included in this study as they were not reliably measured in all studies and measurement can be positional. Additionally, we have noted that the A wave reversal can be absent in severe restriction only becoming obvious after relief of the obstruction which may be to do with reduced pulmonary flow in the face of obstruction.

The Doppler of the left pulmonary artery band appears to be the most affected by increasing atrial restriction. The left pulmonary artery often falls in a better plane for Doppler interrogation compared to the right and therefore Doppler traces may be more accurate. It may also relate to the left pulmonary artery band being generally looser. Access to the left pulmonary artery can be challenging (a short intra-pericardial length)(8) both during HP and when it comes to pulmonary artery reconstruction at the subsequent stage. A looser band may therefore be more representative of acute changes in the pulmonary vascular bed, although it should be noted that a similar waveform is seen when the band is too loose.

As the HP population is small, there are a relatively small number of patients in this cohort which did not therefore allow comparison between the groups with and without forward flow. Not all measurements were available for all scans at all time-points, and some (albeit few) scans were performed $>48$ hours after the procedure. Additionally, some patients had the atrial septostomy as a proactive or opportunistic intervention, when another intervention was performed. Invasive catheter data was not available for all patients as many had septostomies performed under echocardiographic guidance only, if there had been more invasive data, we may have been able to correlate this with some of the echocardiographic parameters.

\section{Conclusion}

Serial echocardiography is important in assessing atrial restriction in patients with HLHS after the hybrid procedure. Along with measurements of the transatrial gradient and pulmonary venous Doppler interrogation (which may be affected by pulmonary flow status), Doppler interrogation of the left pulmonary band may allow further confirmation of important atrial restriction.

\section{Acknowledgments}

We would like to thank the staff of the echocardiography and paediatric cardiology department at Evelina London Children's Hospital.

\section{References}


1. Gibbs JL, Wren C, Watterson KG, Hunter S, Hamilton JR Stenting of the arterial duct combined with banding of the pulmonary arteries and atrial septectomy or septostomy: a new approach to palliation for the hypoplastic left heart syndrome. Br Heart J 1993;69(6):551-5.

2. Gutgesell HP, Lim DS Hybrid palliation in hypoplastic left heart syndrome. Current Opinion in Cardiology 2007;22(2):55-9. Doi: 10.1097/HCO.0b013e328014d945.

3. Pizarro C, Davies RR, Woodford E, Radtke WA Improving early outcomes following hybrid procedure for patients with single ventricle and systemic outflow obstruction: defining risk factors. European Journal of Cardio-Thoracic Surgery 2015;47(6):995-1001. Doi: 10.1093/ejcts/ezu373.

4. Honjo O, Benson LN, Mewhort HE, et al. Clinical Outcomes, Program Evolution, and Pulmonary Artery Growth in Single Ventricle Palliation Using Hybrid and Norwood Palliative Strategies 2009;87(6):1885-93. Doi: 10.1016/j.athoracsur.2009.03.061.

5. Fenstermaker B, Berger GE, Rowland DG, et al. Interstage Echocardiographic Changes in Patients Undergoing Hybrid Stage I Palliation for Hypoplastic Left Heart Syndrome. Journal of the American Society of Echocardiography 2008;21(11):1222-8. Doi: 10.1016/j.echo.2008.08.005.

6. Chintala K, Tian Z, Du W, Donaghue D, Rychik J Fetal pulmonary venous Doppler patterns in hypoplastic left heart syndrome: relationship to atrial septal restriction. Heart 2008;94(11):1446-9. Doi: 10.1136/hrt.2007.123497.

7. MD SME, MD EAB, Doff B McElhinney MD M, et al. Primary left ventricular rehabilitation is effective in maintaining two-ventricle physiology in the borderline left heart. The Journal of Thoracic and Cardiovascular Surgery 2009;138(6):1276-82. Doi: 10.1016/j.jtcvs.2009.08.009.

8. Pizarro C, Derby CD, Radtke WA Hybrid Procedure for Hypoplastic Left Heart Syndrome: The Nemours Approach. Yotct 2015;14(2):86-98. Doi: 10.1053/j.optechstcvs.2009.05.002.

Tables

Table 1. Characteristics of the two groups

Table 1. Characteristics of the two groups

Male Classical HLHS Antenatal diagnosis Indication Weight Clinical condition^ Borderline Mean (SD) weight M Hypoplastic left heart syndrome (HLHS), hybrid procedure (HP), standard deviation (SD) ^clinical condition was defined a

Table 2. Septostomy Group

Table 2. Septostomy Group

Mean IAS velocity, m/s IAS VTI, cm RPA S, m/s* RPA D, m/s*^ RPA mean, m/s RPA VTI, cm RPA S vel:D vel*^ RPA $\mathrm{p}<0.05$ looking at change over time ${ }^{\wedge} \mathrm{p}<0.05$ looking at differences between RPA and LPA Dopplers Interatrial atrial septur

Table 3. Model of Change

Table 3. Model of Change

Peak S velocity Diastolic velocity Mean velocity VTI S vel: D vel ratio Pulsatility index Diastolic (D), systolic (S), velocity time integral (VTI) Numbers represent P values for three components. "Change over tir

Table 4. Controls versus septostomy post-hybrid 
Table 4. Controls versus septostomy pre-septostomy

Mean IAS velocity, m/s IAS VTI, cm RPA S, m/s RPA D, m/s RPA mean, m/s RPA VTI, cm RPA S vel:D vel RPA PI L $\mathrm{p}<0.05$ Interatrial atrial septum (IAS), left pulmonary artery (LPA), pulsatility index (PI), right pulmonary artery (RPA),

\section{Figures}

Figure 1 Tracing of the transatrial Doppler over two cardiac cycles

Figure 2 Tracing of the right pulmonary artery band Doppler (red) with measurement of the peak systolic velocity (green) and diastolic velocity (blue)

Figure 3 Left pulmonary artery band Doppler pre-atrial intervention showing low diastolic and notching in two patients $(\mathrm{a}, \mathrm{b})$ and a schematic (c). Left pulmonary artery band Doppler post-atrial intervention showing higher diastolic pressure and less prominent notching in the same two patients (d,e) and a schematic

Figure 4 The left and right pulmonary artery band parameters over time 


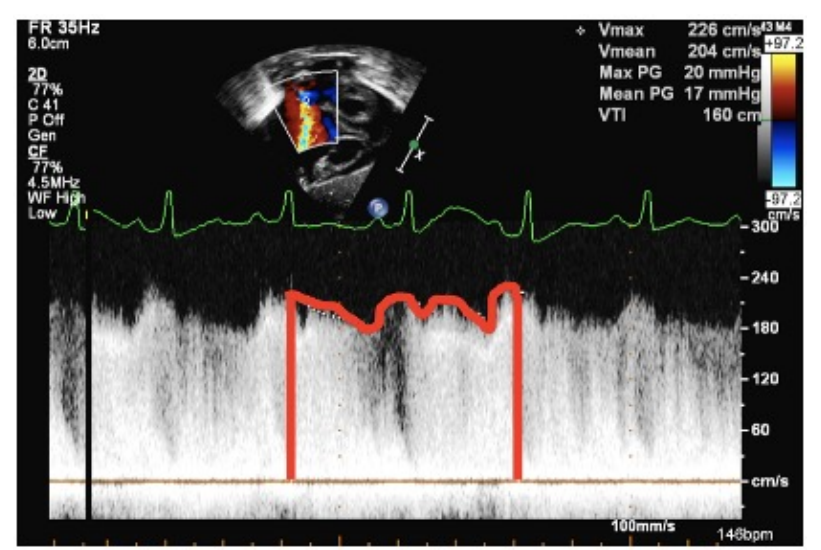




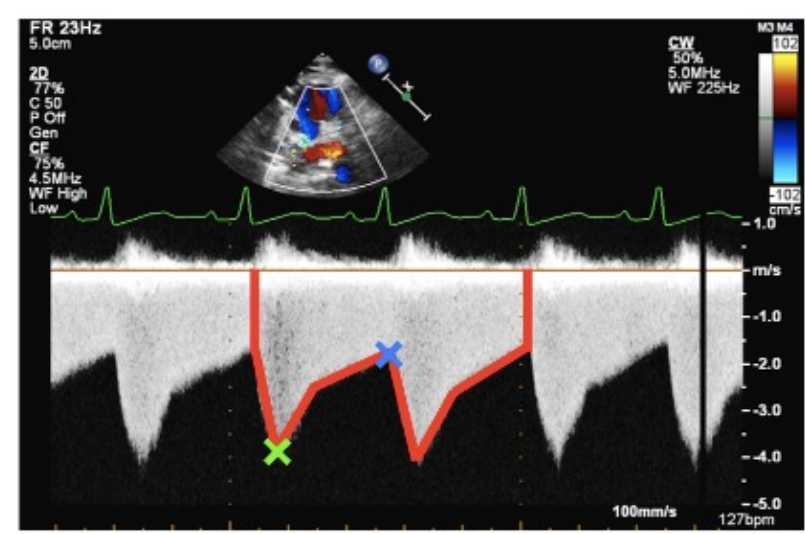




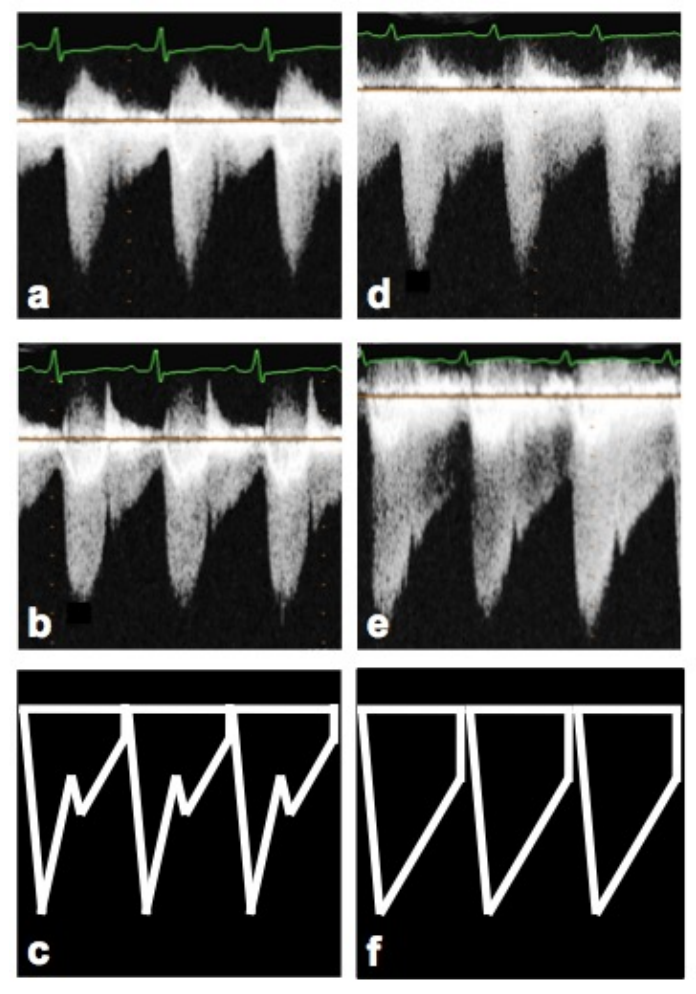



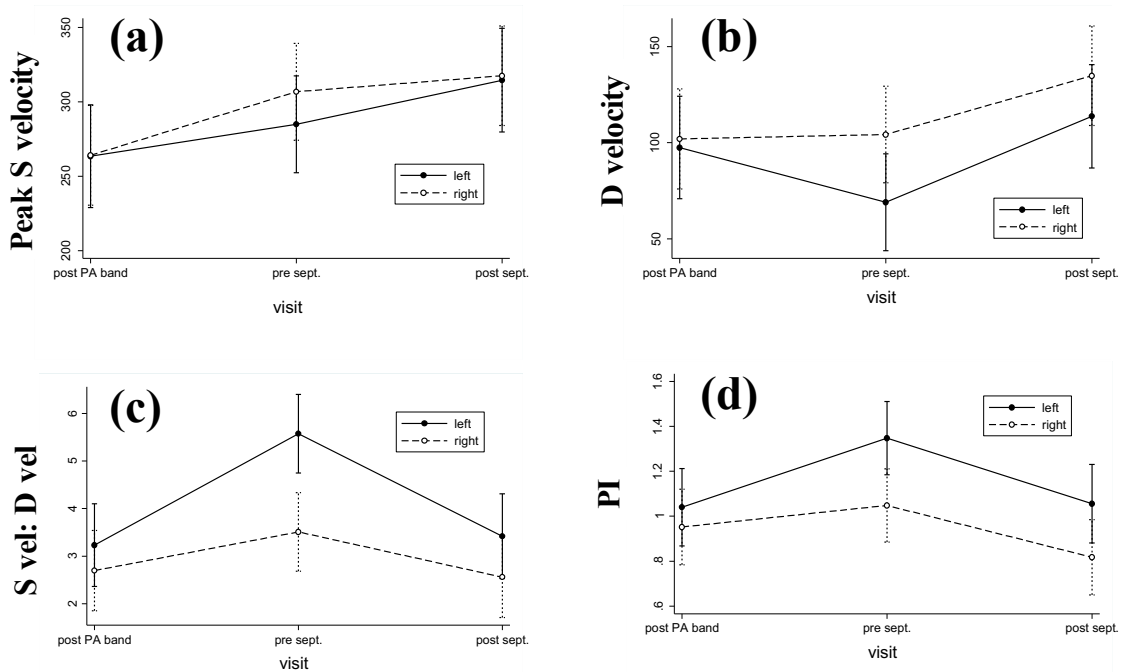